\title{
The use of a clinical decision support tool to assess the risk of QT drug-drug interactions in community pharmacies
}

\author{
Florine A. Berger ${ }^{\text {iD }}$, Heleen van der Sijs, Teun van Gelder and \\ Patricia M. L. A. van den Bemt
}

\begin{abstract}
Introduction: The handling of drug-drug interactions regarding QTc-prolongation (QT-DDIs) is not well defined. A clinical decision support (CDS) tool will support risk management of QTDDIs. Therefore, we studied the effect of a CDS tool on the proportion of QT-DDIs for which an intervention was considered by pharmacists.

Methods: An intervention study was performed using a pre- and post-design in 20 community pharmacies in The Netherlands. All QT-DDIs that occurred during a before- and after-period of three months were included. The impact of the use of a CDS tool to support the handling of QT-DDIs was studied. For each QT-DDI, handling of the QT-DDI and patient characteristics were extracted from the pharmacy information system. Primary outcome was the proportion of QT-DDIs with an intervention. Secondary outcomes were the type of interventions and the time associated with handling QT-DDIs. Logistic regression analysis was used to analyse the primary outcome.

Results: Two hundred and forty-four QT-DDIs pre-CDS tool and 157 QT-DDIs post-CDS tool were included. Pharmacists intervened in $43.0 \%$ and $35.7 \%$ of the QT-DDIs pre- and postCDS tool respectively lodds ratio $0.74 ; 95 \%$ confidence interval $0.49-1.11$ ). Substitution of interacting agents was the most frequent intervention. Pharmacists spent $20.8 \pm 3.5 \mathrm{~min}$ (mean $\pm \mathrm{SD}$ ) on handling QT-DDIs pre-CDS tool, which was reduced to $14.9 \pm 2.4 \mathrm{~min}$ (mean \pm SD) post-CDS tool. Of these, $4.5 \pm 0.7$ min (mean \pm SD) were spent on the CDS tool. Conclusion: The CDS tool might be a first step to developing a tool to manage QT-DDIs via a structured approach. Improvement of the tool is needed in order to increase its diagnostic value and reduce redundant QT-DDI alerts.
\end{abstract}

Keywords: clinical decision support systems, community pharmacies, drug-drug interactions, intervention, primary care

Received: 27 November 2019; revised manuscript accepted: 22 January 2021.

\section{Plain Language Summary}

The use of a tool to support the handling of QTc-prolonging drug interactions in community pharmacies

Introduction: Several drugs have the ability to cause heart rhythm disturbances as a rare side effect. This rhythm disturbance is called QTc-interval prolongation. It may result in cardiac arrest. For health care professionals, such as physicians and pharmacists, it is difficult to decide whether or not it is safe to proceed treating a patient with combinations

\footnotetext{
Correspondence to: Florine A. Berger Department of Hospital Pharmacy, Erasmus University Medical Centre, Department of Hospital Pharmacy, Doctor Molewaterplein 40, 3015 GD Rotterdam, The Netherlands f.bergerderasmusmc.nl Heleen van der Sijs Department of Hospital Pharmacy, Erasmus University Medical Centre, Rotterdam, The Netherlands

Teun van Gelder Department of Hospital Pharmacy, Erasmus University Medical Centre, Rotterdam, The Netherlands

Department of Clinical Pharmacy and Toxicology, Leiden University Medical Centre, Leiden, The Netherlands

Patricia M. L. A. van den Bemt

Department of Hospital

Pharmacy, Erasmus University Medical Centre, Rotterdam, The Netherlands

Department of

Clinical Pharmacy and Pharmacology, University Medical Centre Groningen, Groningen, The Netherlands
} 
of two or more of these QT-prolonging drugs. Recently, a tool was developed that supports the risk management of these QT drug-drug interactions (QT-DDIs).

Methods: In this study, we studied the effect of this tool on the proportion of QT-DDIs for which an intervention was considered by pharmacists. An intervention study was performed using a pre- and post-design in 20 community pharmacies in The Netherlands. All QT-DDIs that occurred during a before- and after-period of 3 months were included. Results: Two hundred and forty-four QT-DDIs pre-implementation of the tool and 157 QTDDIs post-implementation of the tool were included. Pharmacists intervened in $43.0 \%$ of the QT-DDIs before the tool was implemented and in $35.7 \%$ after implementation of the tool. Substitution of one of the interacting agents was the most frequent intervention. Pharmacists spent less time on handling QT-DDIs when the tool was used.

Conclusion: The clinical decision support tool might be a first step to developing a tool to manage QT-DDIs via a structured approach.

\section{Introduction}

Drug-drug interactions (DDIs) regarding QTcprolongation are common in daily practice due to the high number of drugs known for prolonging the QTc-interval. Currently, over 50 drugs are associated with causing Torsade de Points (TdP) by prolonging the QTc-interval, according to the CredibleMeds $^{\circledR}$ QT-drug lists of the Arizona Center for Education and Research on Therapeutics (AzCERT). ${ }^{1}$ This number has been increasing over the years as new drugs are added to the QT-drug list due to monthly reviews of AzCERT. ${ }^{2}$ QTc-prolongation is used as a surrogate marker for the risk of TdP, a ventricular tachycardia which may ultimately lead to ventricular fibrillation or sudden cardiac death. ${ }^{3-5}$ Although QTc-prolongation is not the perfect marker for arrhythmia risk as many other risk factors play a role in developing $\mathrm{TdP}$, it has become the primary safety parameter among health care professionals, because it is still the most validated marker for the proarrhythmic potency of drugs. ${ }^{5-7}$

Although a QTc-prolonging drug in itself will rarely induce clinically relevant QTc-prolongation $(>500 \mathrm{~ms}$ ), a combination of QTc-prolonging drugs in patients with multiple risk factors can result in QTc-intervals above $500 \mathrm{~ms} .{ }^{8,9}$

The Dutch drug database 'G-standard', which contains information for clinical decision support, describes the current guidelines for risk management of drug safety alerts. In The Netherlands, clinical decision support (CDS) systems in primary and secondary care generate QT-DDI alerts when two QTc-prolonging drugs with a known risk of TdP are combined. More than $40 \%$ of the processed drug prescriptions lead to drug safety alerts. ${ }^{10}$ However, the specificity of the alerts generated by CDS systems is very low, resulting in a low number of interventions. At the moment, there is a complete lack of discrimination when handling these QT-DDIs. Many of the generated QT-DDI alerts do not require an intervention. With the increasing number of QTc-prolonging drugs, alert fatigue could be imposed on physicians. Low specificity alerts contribute to non-compliance with current guidelines. ${ }^{11-15}$ To decrease the alert burden, more advanced clinical rules including clinical parameters such as patient characteristics and laboratory values are used to improve the specificity of the alerts and decrease the alert rate. ${ }^{16-20}$

In the case of QT-DDI alerts, an advanced clinical rule should be able to discriminate low- and high-risk patients for developing QTcprolongation. QT-DDI alerts are redundant in patients with no other risk factors for QTcprolongation. In high-risk patients, QTcprolonging drugs should be either substituted or routine electrocardiogram (ECG) monitoring is required. This clinical rule should be developed irrespective of the QTc-prolonging drugs involved in the QT-DDIs, due to insufficient data on the absolute effect on QTc-prolongation of these drugs. A risk profile of each individual patient will improve accuracy of QT-DDI alerts and support the risk management of QT-DDIs. ${ }^{8}$

Therefore, a CDS prediction tool was developed to assess the risk of QT-DDIs for developing QTc-prolongation (Figure 1). ${ }^{21,22}$ The main aim of this study was to determine the effect of such a CDS tool on the interventions made by pharmacists in primary care. We also explored the usability of the CDS tool in clinical practice. 


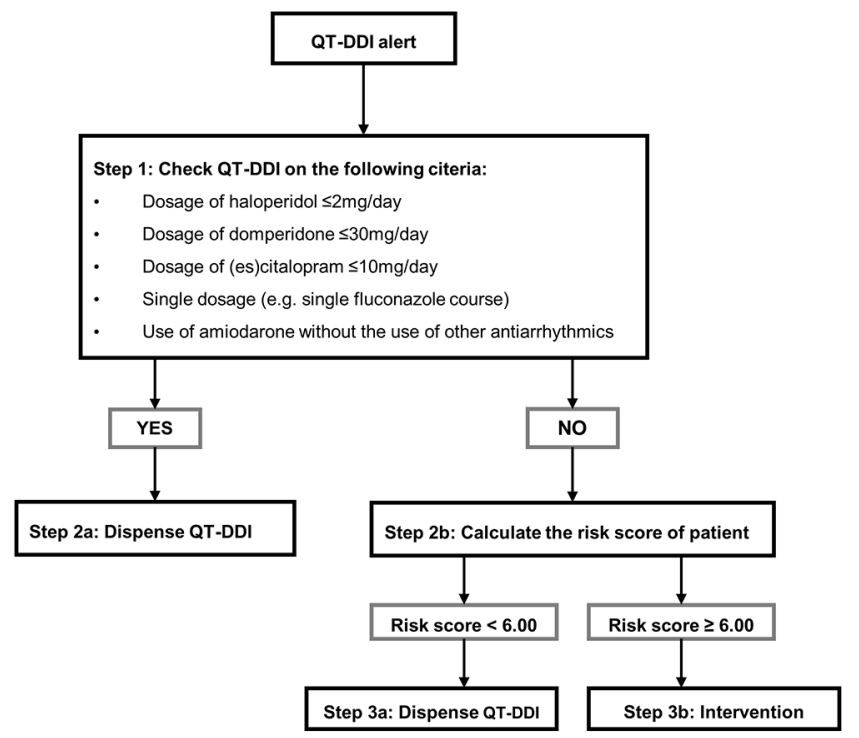

\begin{tabular}{lc}
\hline Risk factors & Score \\
\hline 1. Age (in years) & 1 \\
$\quad 51-75$ & 2 \\
$\quad \geq 76$ & 1 \\
2. Female gender & \\
3. Comorbidities & 2 \\
$\quad$ Cardiovascular comorbidity & 2 \\
$\quad$ Hypertension & 1 \\
$\quad$ Diabetes Mellitus (I and II) & \\
4. Renal function, eGFR (MDRD) & 1 \\
$\quad$ 550 mL min ${ }^{-1}$ & 1 \\
$\quad$ Missing value & \\
5. Potassium serum level & \\
$\quad$ 22,5 mmol L-1 & 2 \\
2.6 - 3.4 mmol L-1 & 1 \\
$\quad$ Missing value & 1 \\
6. Loop diuretics & 2 \\
7. QTc-prolonging drugs & \\
\hline
\end{tabular}

Figure 1. Clinical decision support tool to assess the risk of QT-DDIs. QTc-prolonging drugs with a known risk of $\mathrm{TdP}^{1}$

eGFR, estimated glomerular filtration rate; MDRD, Modification of Diet in Renal Disease; QT-DDI, QT drug-drug interaction.

\section{Methods}

\section{Development of a prediction model}

This CDS prediction model was developed by performing a prospective observational study in 107 patients using two or more QTc-prolonging drugs with a known risk of $\mathrm{TdP}^{1}$ to identify risk factors for QTc-prolongation. ${ }^{21}$ A standard 12-lead resting ECG was recorded at the estimated time of peak concentration $\left(T_{\max }\right)$ of the last added drug, or at the longest $T_{\max }$ in the case of both drugs being started at the same time. Risk factors were identified using logistic regression analyses and risk points were assigned based on the odds ratios. Additional risk factors were incorporated into the model based on a literature review on risk factors for QTc-prolongation. The CDS tool was validated in an external dataset $(n=8,453)$, resulting in an area under the receiver operating characteristic-curve of 0.59 (95\% CI 0.54-0.63) when QTc-prolongation was defined as $>500 \mathrm{~ms}$ due to many false positive results. The selected optimal cut-off value was $6 ; 26.3 \%$ of all patients scored $<6$ points. A sensitivity of $83.9 \%$ and a specificity of $27.5 \%$ were accomplished with a cut-off value of 6 . The discriminative ability of the tool is not perfect, so optimization of the tool is required. ${ }^{22}$ On the other hand, there is currently a complete lack of discrimination when handling these QT-DDIs. There should be a balance between the number of alerts generated by the CDS systems and its effect on patient care. Although not perfect, this tool is still able to reduce the number of redundant QT-DDI alerts.

\section{Study design}

An intervention study was performed using a pre- and post-design in 20 community pharmacies in The Netherlands. We implemented the use of the CDS tool (consisting a paper-based flowchart) to study the impact on the handling of QT-DDIs.

All QT-DDIs that occurred during a pre- and post-CDS tool period of 3 months were included. The QTc-prolonging drugs involved in the QT-DDIs are listed at the CredibleMeds ${ }^{\circledR}$ QT-drug list with a known risk of TdP (Supplemental material Table S1 online). Only pharmacies using the pharmacy information system Pharmacom ${ }^{\circledR}$ (by TSS Pharma Partners) in the region of Rotterdam (Rijnmond) were included to ensure conformity in data capture and data extraction. The medical ethics review board of the Erasmus University Medical Centre approved the protocol and waived the requirement for obtaining 
informed consent (MEC-2015-513). The study was conducted according to the principles of the Declaration of Helsinki.

\section{Study population}

All QT-DDIs including QTc-prolonging drugs with a known risk of TdP that occurred in the community pharmacies during a study period of 3 months before and 3 months after implementation of the CDS tool were included. QT-DDIs of patients younger than 18 years old were excluded.

\section{Outcome measures}

The primary outcome measure of this study was the proportion of QT-DDIs in which pharmacists intervened. An intervention was defined as a consultation with prescribers to discuss the clinical relevance of the QT-DDI, and proposal for further actions to be taken (hereafter referred to as intervention). Secondary outcome measures were the types of interventions made by pharmacists. The interventions for the QT-DDIs were subsequently categorized in (I) dispensing both drugs on account of the prescriber, (II) ECG monitoring, (III) substitution of one of the interacting agents, (IV) dose adjustments or (V) (temporarily) stopping one of the interacting agents. We have also studied the difference between firsttime prescriptions and repeat-prescriptions on the handling and types of interventions. Another secondary outcome measure was the time spent on handling QT-DDIs. Finally, the CDS tool was evaluated by pharmacists on usability in clinical practice.

\section{Data collection}

For all QT-DDIs, the following variables were collected: the management of the QT-DDI including interventions, the interacting drugs and the dosages of interacting drugs.

For all patients: age, gender, comorbidities (registered as drug-disease interactions) were collected. Concomitant drug use was retrieved from the medication history up to 1 year prior to the QT-DDI alert. The following laboratory values were collected, if registered in Pharmacom ${ }^{\circledR}$ : renal function, liver function parameters and electrolyte serum levels. Patient data were handled confidentially and were extracted anonymously according to the Dutch Personal Data Protection Act (Wbp). All patients with QT-DDI alerts were captured in an electronic clinical data management system (OpenClinica, LLC, Waltham, United States).

\section{CDS tool}

The CDS tool was implemented in the participating community pharmacies after a 3 month baseline analysis as a tool to support the electronic handling of QT-DDI alerts. The CDS tool consisted of a paper-based flowchart identifying patients that were at increased risk for developing QTc-prolongation as is shown in Figure 1. The criteria of step 1 of the flowchart were based on a literature review and an expert panel with two cardiologists with expertise in electrophysiology and publications in the field. ${ }^{23-30}$ Before implementation, the pharmacists were trained in using the CDS tool. The CDS tool was expected to be used for all QT-DDI alerts during the post-implementation period. When patients scored $\geqslant 6$ using the tool, an intervention by pharmacists was recommended. The risk scores and types of interventions were documented using a paper form.

\section{Usability clinical decision support tool}

After a period of 3 months, the tool was evaluated by the pharmacists on usability in clinical practice using the System Usability Scale (SUS) of Brooke. ${ }^{28,29}$ The SUS is based on 3 usability measures suggested by the International Organization for Standardization (ISO)-9241-11: effectiveness, efficiency and satisfaction. The SUS consists of a 10-item questionnaire covering subjective items of usability using a five-point Likert scale with a degree from total disagreement (1) to total agreement (5). For evaluation of the CDS tool, 10 items were formulated so that they were compatible for the CDS tool (Supplemental Table S2). Items 1, 3, 6, 7 and 9 were positively formulated and items 2, 4, 5, 8 and 10 were negatively formulated. The SUS score was calculated, first by recalculating the score of each item (1-5) to a range from $0-4$ using the following formula; for the positively formulated items: scale position minus 1 , and for the negatively formulated items: 5 minus scale position. Second, the sum of these scores was multiplied by 2.5 to obtain the overall SUS score. ${ }^{31}$ Total SUS scores range from 0 to 100 ; if SUS scores are $<60$ the system is considered to be unacceptable, $60-70$ is acceptable, 
$70-80$ is good, $80-90$ is very good and $>90$ is excellent. ${ }^{32,33}$

\section{Statistical analysis}

Based on information provided by the Stevens Institute for Research (SIR) in Leiden, The Netherlands, Dutch community pharmacies on average dispense QTc-prolonging drugs 140 times a year with a QT-DDI (interaction code 6297). That results in 0.5 QT-DDIs per day per pharmacy in which the DDI-causing drug is dispensed. A QT-DDI does not always result in dispensing, so we estimate that one QT-DDI per day per pharmacy will be handled. So, in 3 months (pre and post measurement) approximately 45 QT-DDIs per pharmacy were expected to be monitored, which would result in a total number of 900 QT-DDIs in 20 community pharmacies. With an alpha of 0.05 and a power of $80 \%$, a difference in proportion of QT-DDIs with intervention of $60 \%$ (pre) versus $66 \%$ (post) can be established using logistic regression, estimating that in $60 \%$ of the QT-DDIs an intervention is carried out.

The primary outcome was determined by dividing the number of QT-DDIs with an intervention by the total number of QT-DDIs. Univariate logistic regression analysis was used to analyse the primary outcome between the measurements before and after implementation of the CDS tool. If a variation in interaction characteristics occurred between the before- and after-period, the primary outcome was adjusted using multivariate logistic regression analysis. Odds ratios (ORs) and their 95\% confidence intervals (CIs) were reported. Secondary outcome measures were analysed using descriptive statistics.

\section{Results}

\section{Study population}

The baseline characteristics of the participating community pharmacies are presented in Table 1 . The after-period included 16 community pharmacies because four pharmacists, and therefore four pharmacies, discontinued their participation because of construction work of the pharmacy and shortages in personnel. A total of 928 QT-DDI alerts were generated during the preand post-CDS tool phases, of which 401
Table 1. Baseline characteristics of the participating community pharmacies.

\begin{tabular}{|c|c|}
\hline Pharmacy characteristics & $\begin{array}{l}\text { Cohort } \\
N=20\end{array}$ \\
\hline FTE pharmacists, mean \pm SD & $1.7 \pm 0.9$ \\
\hline FTE pharmacy assistants, mean \pm SD & $7.0 \pm 3.3$ \\
\hline \multicolumn{2}{|l|}{ HKZ certificates, $n(\%)$} \\
\hline None & - \\
\hline Chain certificate & $7(35)$ \\
\hline Own certificate & $13(65)$ \\
\hline \multicolumn{2}{|l|}{ Collaboration with other community pharmacies, $n(\%)$} \\
\hline None & $8(40)$ \\
\hline$<5$ & $4(20)$ \\
\hline $5-25$ & $3(15)$ \\
\hline$>25$ & $5(25)$ \\
\hline \multicolumn{2}{|l|}{ GPs responsible for $>80 \%$ of prescriptions, $n(\%)$} \\
\hline $1-3$ & $5(25)$ \\
\hline $4-6$ & $10(50)$ \\
\hline $7-9$ & $3(15)$ \\
\hline$\geqslant 10$ & $2(10)$ \\
\hline Shared patient records with GP, $n(\%)$ & $14(70)$ \\
\hline Community Health Centre, $n(\%)$ & $12(60)$ \\
\hline Use of EPR in Pharmacom ${ }^{\circledR}, n(\%)$ & $18(90)$ \\
\hline$\%$ of renal function parameters available, mean \pm SD & $71.7 \pm 20.9$ \\
\hline$\%$ of potassium serum levels available, mean \pm SD & $58.1 \pm 31.9$ \\
\hline$\%$ of shared contra-indications with $\mathrm{GP}$, mean $\pm \mathrm{SD}$ & $77.2 \pm 19.7$ \\
\hline
\end{tabular}

QT-DDIs were included for analysis. In the before-period, 244 QT-DDIs belonging to 233 patients were included for analysis. Six QT-DDIs were excluded because they occurred in patients $<18$ years old (Figure 2). In the after-period, a total of 157 QT-DDIs of 149 patients were included, as shown in Figure 3. Only 23 patients were included in both the 
Table 2. Baseline characteristics of study population.

\begin{tabular}{|c|c|c|c|}
\hline \multirow[t]{2}{*}{ Patient characteristics } & \multirow{2}{*}{$\begin{array}{l}\text { Before-period } \\
n=233\end{array}$} & \multirow{2}{*}{$\frac{\text { After-period }}{n=149}$} & \multirow[t]{2}{*}{$p$-value } \\
\hline & & & \\
\hline Age, years, median; IQR & $66.0 ; 26.0$ & $63.0 ; 31.0$ & $0.86^{\mathrm{a}}$ \\
\hline$\leqslant 50, n(\%)$ & $60(25.8)$ & $41(27.5)$ & $0.72^{\mathrm{b}}$ \\
\hline $51-75, n(\%)$ & $113(48.5)$ & $66(44.3)$ & \\
\hline$\geqslant 76, n[\%]$ & $60(25.8)$ & $42(28.2)$ & \\
\hline Female gender, $n(\%)$ & $154(66.1)$ & $91(61.1)$ & $0.32^{b}$ \\
\hline BMI, median; IQR & $31.9(n=1)$ & $24.5(n=1)$ & - \\
\hline \multicolumn{4}{|l|}{ Comorbidities, $n(\%)$} \\
\hline Myocardial infarction & $25(10.7)$ & $17(11.4)$ & $0.84^{b}$ \\
\hline Heart failure & $13(5.6)$ & $2(1.3)$ & $0.06^{c}$ \\
\hline Arrhythmia & $14(6.0)$ & $3(2.0)$ & $0.08^{c}$ \\
\hline Hypertension & $68(29.2)$ & $43(28.9)$ & $0.95^{\mathrm{b}}$ \\
\hline Diabetes mellitus & $39(16.7)$ & 29 (19.5) & $0.50^{\mathrm{b}}$ \\
\hline COPD/asthma & $42(18.0)$ & $23(15.4)$ & $0.51^{b}$ \\
\hline CVA/TIA & $5(2.1)$ & $8(5.4)$ & $0.09 \mathrm{~b}$ \\
\hline Renal dysfunction & $16(6.9)$ & $12(8.1)$ & $0.66^{\mathrm{b}}$ \\
\hline Liver dysfunction & $9(3.9)$ & $4(2.7)$ & $0.77^{c}$ \\
\hline Others & $111(47.6)$ & $74(49.7)$ & $0.70^{\mathrm{b}}$ \\
\hline \multicolumn{4}{|c|}{ Renal dysfunction with renal function, $n(\%)$} \\
\hline eGFR, MDRD $\leqslant 50 \mathrm{ml} / \mathrm{min}$ & $11(4.7)(n=20)$ & $10(6.7)(n=19)$ & $0.97 \mathrm{~b}$ \\
\hline \multicolumn{4}{|l|}{ Electrolyte disturbances, $n(\%)$} \\
\hline Hyponatraemia $\left(\mathrm{Na}^{+}<136 \mathrm{mmol} / \mathrm{l}\right)$ & $-(n=1)$ & $-(n=2)$ & - \\
\hline Hypokalaemia $\left(\mathrm{K}^{+}<3.5 \mathrm{mmol} / \mathrm{l}\right)$ & $-(n=1)$ & $-(n=2)$ & - \\
\hline \multicolumn{4}{|c|}{$\begin{array}{l}\text { alndependent } t \text { test. } \\
\text { 'Chi-square test. } \\
\text { cFisher's exact test. } \\
\text { BMI, body mass index; COPD, chronic obstructive pulmonary disease; CVA, cerebrovascular accident; eGFR, estimated } \\
\text { glomerular filtration rate; IQR, interquartile range; MDRD, Modification of Diet in Renal Disease; TIA, transient ischemic } \\
\text { attack. }\end{array}$} \\
\hline
\end{tabular}

before- and after-periods; in 17 patients the QT-DDIs were identical.

The baseline patient characteristics of patients in the before- and after-periods are shown in Table 2. The two groups did not significantly differ in patient characteristics. The median (interquartile range) age of the total cohort was 65 (28) years and most QT-DDI alerts belonged to female patients $(64.1 \%)$. From only a limited numbers of patients the renal function parameters $(10.2 \%)$ and potassium levels $(0.8 \%)$ could 


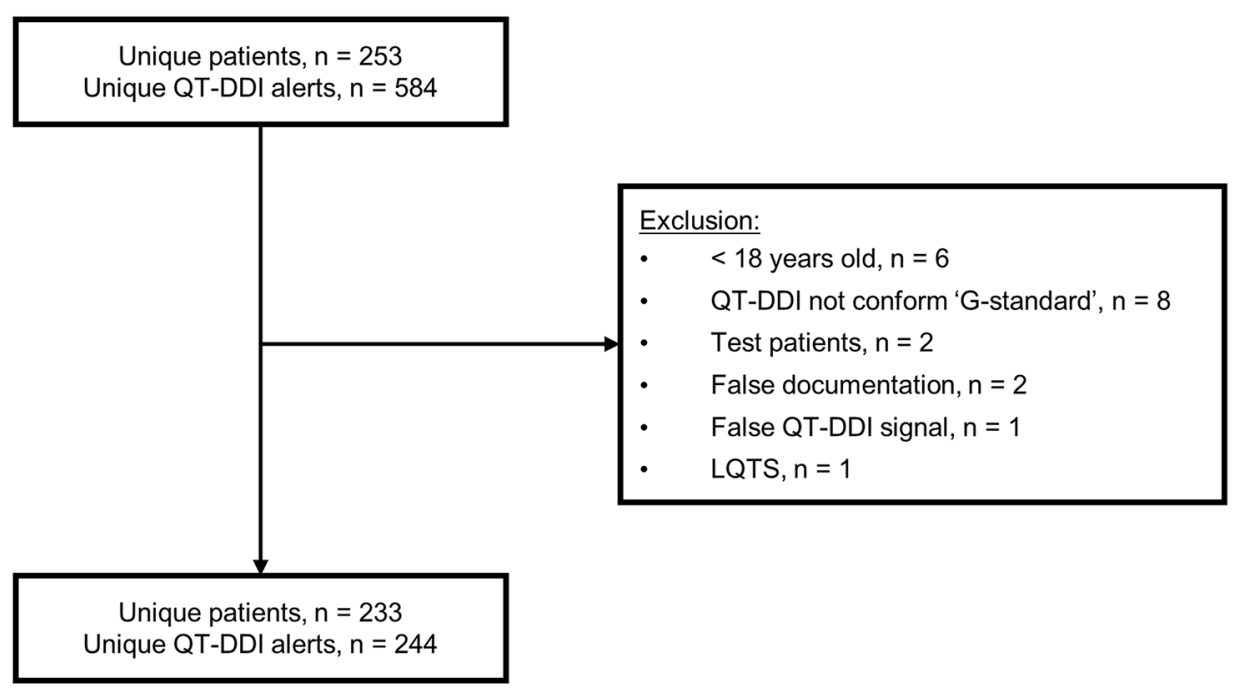

Figure 2. Flowchart of QT-DDI inclusions in before-period. LQTS, long QT syndrome; QT-DDI, QT drug-drug interaction.

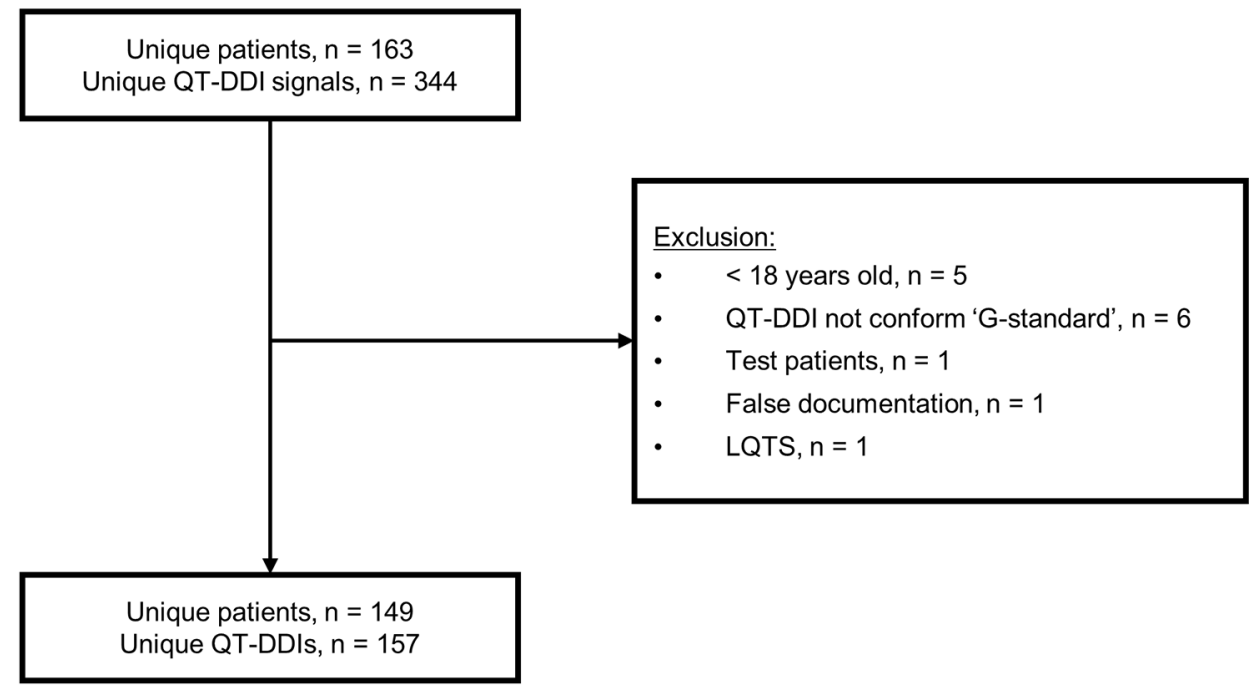

Figure 3. Flowchart of QT-DDI inclusions in after-period. LQTS, long QT syndrome; QT-DDI, QT drug-drug interaction.

be extracted from Pharmacom ${ }^{\circledR}$. In clinical practice, pharmacy information systems are frequently linked to the information system of the general practitioners (GPs). Unfortunately, it was not possible to retrieve these variables from the GP information system due to privacy legislation.

There was no significant difference in the proportion of first-time prescriptions in the QT-DDIs in the before-period $(62.5 \%)$ compared with the after-period $(73.9 \%, p=0.08)$. In both periods, the QT-DDI that occurred most frequently was (es)citalopram - haloperidol $(10.8 \%$ and $10.7 \%$, respectively). The top-10 QT-DDIs and QTcprolonging drugs are presented in Table 3 . When drug groups were classified, the most common QT-DDI was a QTc-prolonging drug combined with an antiarrhythmic agent class III $(34.0 \%)$ in 
Table 3. Top-10 QT-DDIs.

\begin{tabular}{|c|c|c|}
\hline \multirow[t]{2}{*}{ QT-DDIs } & Before- & After- \\
\hline & $n=244(\%)$ & $n=157(\%)$ \\
\hline $\begin{array}{l}\text { 1. Haloperidol - (es) } \\
\text { citalopram }\end{array}$ & $26(10.7)$ & $17(10.8)$ \\
\hline $\begin{array}{l}\text { 2. Amiodarone - } \\
\text { ciprofloxacin }\end{array}$ & 13 (5.3) & $7(4.5)$ \\
\hline $\begin{array}{l}\text { 3. Azithromycin - (es) } \\
\text { citalopram }\end{array}$ & $12(4.9)$ & 13 (8.3) \\
\hline $\begin{array}{l}\text { 4. (Es)citalopram - } \\
\text { fluconazole }\end{array}$ & $11(4.5)$ & 5 (3.2) \\
\hline $\begin{array}{l}\text { 5. Azithromycin - } \\
\text { domperidone }\end{array}$ & $10(4.1)$ & 2 (1.3) \\
\hline $\begin{array}{l}\text { 6. Sotalol - } \\
\text { ciprofloxacin }\end{array}$ & $10(4.1)$ & 5 (3.2) \\
\hline $\begin{array}{l}\text { 7. Haloperidol - } \\
\text { ciprofloxacin }\end{array}$ & 9 (3.7) & $7(4.5)$ \\
\hline 8. Sotalol - flecainide & 8 (3.3) & $6(3.8)$ \\
\hline $\begin{array}{l}\text { 9. Sotalol - (es) } \\
\text { citalopram }\end{array}$ & 7 (2.9) & - \\
\hline $\begin{array}{l}\text { 10. Azithromycin - } \\
\text { fluconazole }\end{array}$ & $6(2.4)$ & $10(6.4)$ \\
\hline
\end{tabular}

the before-period and a QTc-prolonging drug combined with a QTc-prolonging antibiotic $(32.5 \%)$ in the after-period. The mean \pm SD number of QT-DDIs per pharmacy was $12.2 \pm 7.6$ in the before-period and $9.8 \pm 5.1$ in the after-period.

\section{Outcome measures}

There was no significant difference in the proportion of QT-DDIs for which an intervention was made after implementing the CDS tool; $43.0 \%$ before and $35.7 \%$ after implementation (OR $0.74,95 \%$ CI $0.49-1.11 ; p=0.14$ ). A sensitivity analysis, performed by excluding 23 patients who were included in both the pre- and the post-period, had no effect on the significance of the primary outcome (OR $0.78,95 \%$ CI 0.50 $1.21 ; p=0.27)$.
An interacting agent was substituted in $41.0 \%$ of the interventions in the before-period and in $46.4 \%$ in the after-period. In $37.7 \%$ of the QT-DDIs with intervention, the QTc-prolonging drugs were dispensed in the before-period, and in $26.8 \%$ of the QT-DDIs in the after-period. Almost $12 \%$ of all alerts were incorrect QT-alerts because one of the interacting agents was already stopped or the combination was not given simultaneously. In $5.2 \%$ of the QT-DDIs, pharmacists did not properly document their actions. The variety of interventions performed by pharmacists are presented in Table 4 .

After implementation, 157 QT-DDI alerts were handled electronically. However, pharmacists completed the paper forms in only $30.6 \%$ of the QT-DDI alerts. Of these, 30 QT-DDIs (63.5\%) could be dispensed without intervention according to the flowchart of the CDS tool. Pharmacists dispensed the QT-DDI with no intervention, and therefore adhered to the flowchart in 19 cases $(63.3 \%)$. In the remaining cases $(n=11)$, prescribers were consulted. Note that six $(54.5 \%)$ of these cases included amiodarone, of which five $(83.3 \%)$ had a risk score $\geqslant 6$; in these cases, an intervention was performed. Of the 18 QT-DDIs where the flowchart advised to score patients using the risk model, four patients scored $<6$; these QT-DDIs were dispensed without intervention. In all patients with a risk score $\geqslant 6$, prescribers were consulted and in $92.8 \%$ an intervention was performed. Taking all QT-DDIs into account, the overall compliance with the CDS tool by pharmacists was $75 \%$.

When we focus on the criteria of step 1 of the flowchart, the proportion of interventions when QT-DDIs included amiodarone did not differ between the two periods $(48.1 \%$ versus $47.6 \%$; $p=0.97)$. The proportion of interventions decreased in QT-DDIs including (es)citalopram $\leqslant 10 \mathrm{mg} \quad(42.3 \%$ versus $17.3 \% ; p=0.07)$, domperidone $\leqslant 30 \mathrm{mg} \quad(45.9 \%$ versus $9.1 \%$; $p=0.03)$ and haloperidol $\leqslant 2 \mathrm{mg}(35.7 \%$ versus $20.6 \% ; p=0.15)$. Only in the case of domperidone, the proportion of interventions decreased significantly between the before- and the afterperiod. The difference in types of interventions for QT-DDIs including first-time prescriptions or repeat-prescriptions is shown in Table 5.

Time spent by pharmacists. In the before-period, nine pharmacists documented the time spent on 
Table 4. Type of intervention.

\begin{tabular}{|c|c|c|c|}
\hline \multirow[t]{2}{*}{ Interventions } & \multirow{2}{*}{$\frac{\text { Before-period }}{n=244(\%)}$} & \multirow{2}{*}{$\begin{array}{l}\text { After-period } \\
n=157(\%)\end{array}$} & \multirow[t]{2}{*}{$p$-value } \\
\hline & & & \\
\hline Dispensed on account of prescriber & 30 (12.3) & $12(7.6)$ & \\
\hline Substitution of one of the interacting agents & $43(17.6)$ & $26(16.6)$ & \\
\hline (Temporarily) stopping one of the interacting agents & $23(9.4)$ & $15(9.6)$ & \\
\hline Dose adjustments & $4(1.6)$ & $3(1.9)$ & \\
\hline ECG monitoring & $5(2.0)$ & - & \\
\hline Total & $105(43.0)$ & $56(35.7)$ & 0.14 \\
\hline
\end{tabular}

Table 5. Intervention rate of alerts divided into first-time (FP) and repeat (RP) prescriptions.

\begin{tabular}{|c|c|c|c|c|}
\hline & \multicolumn{2}{|l|}{ Before-period } & \multicolumn{2}{|l|}{ After-period } \\
\hline & Intervention (\%) & No intervention (\%) & Intervention (\%) & No intervention $(\%)$ \\
\hline FP & $87(57.2)$ & $65(42.8)$ & $47(41.2)$ & $67(58.8)$ \\
\hline $\mathrm{RP}$ & $18(19.6)$ & $74(80.4)$ & 9 (20.9) & $34(79.1)$ \\
\hline$p$-value ${ }^{a}$ & $<0.001$ & & 0.018 & \\
\hline
\end{tabular}

the management of QT-DDIs in 56 QT-DDIs. On average, pharmacists spent $21 \mathrm{~min}$ on the management of QT-DDIs, as shown in Table 6. Consultation with the prescriber was the most time-consuming and took $10 \mathrm{~min}$ on average. In five cases the handling of QT-DDIs encompassed more than 1 day, because the pharmacists could not reach the prescriber. Nevertheless, these days were not counted in the overall time as this does not represent the time pharmacists actually spent on QT-DDIs.

After implementation, time management was documented in 48 QT-DDIs (Table 7). Pharmacists selected a variety of QT-DDIs. Based on these QT-DDI, pharmacists spent on average $15 \mathrm{~min}$ on the management of QT-DDIs, approximately $6 \mathrm{~min}$ less than in the beforeperiod. Time spent on consultation with the prescriber was reduced to $4 \mathrm{~min}$. Of these $15 \mathrm{~min}$, nearly $5 \mathrm{~min}$ were spent on completing the CDS flowchart.
Usability CDS prediction tool. The mean \pm SD SUS score of the CDS tool was 74.1 \pm 19.1 (14 pharmacists). The highest (maximum of 4 ) mean score per question was 3.5 and was scored on question 1 regarding satisfaction; pharmacists would like to use the CDS tool in clinical practice. The lowest (minimum of 0 ) mean score per question was scored on question 4 regarding reliability; pharmacists expected to need the support of different literature sources, besides the tool, to safely manage QT-DDIs. Three pharmacists ranked the CDS tool $<60$ and considered the tool as 'unacceptable', mainly because the tool was time-consuming in their opinion. The overall suggestion of most pharmacists was to eventually integrate the clinical decision support tool into the pharmacy information system.

\section{Discussion}

To our knowledge, this was the first study on a CDS tool to support the handling of QT-DDIs 
Table 6. Time for handling QT-DDIs in community pharmacies before implementation of the clinical decision support tool.

\begin{tabular}{|c|c|c|c|c|c|c|}
\hline \multirow[t]{2}{*}{ Time-management QT-DDIs } & \multicolumn{2}{|l|}{$\mathrm{FP}, n=38$} & \multicolumn{2}{|c|}{$\mathrm{RP}, n=12$} & \multicolumn{2}{|c|}{ Discharge, $n=5$} \\
\hline & $n(\%)$ & $\begin{array}{l}\text { Minutes } \\
\text { Mean } \pm \text { SD }\end{array}$ & $n(\%)$ & $\begin{array}{l}\text { Minutes } \\
\text { Mean } \pm \text { SD }\end{array}$ & $n(\%)$ & $\begin{array}{l}\text { Minutes } \\
\text { Mean } \pm \text { SD }\end{array}$ \\
\hline Literature & $19(50.0)$ & $2.6 \pm 4.4$ & $3(25.0)$ & $0.9 \pm 1.7$ & $4(80.0)$ & $10.4 \pm 11.9$ \\
\hline Consult prescriber & $26(68.4)$ & $12.3 \pm 26.8$ & $2(16.7)$ & $1.0 \pm 2.4$ & $3(60.0)$ & $11.0 \pm 16.7$ \\
\hline Consult PA & $18(47.4)$ & $1.8 \pm 2.5$ & $6(50.0)$ & $2.8 \pm 3.5$ & $3(60.0)$ & $2.6 \pm 4.2$ \\
\hline Documentation in PIS & $32(84.2)$ & $2.6 \pm 3.7$ & $7(58.3)$ & $2.8 \pm 3.8$ & $5(100.0)$ & $5.8 \pm 4, .1$ \\
\hline Consult patient & $23(60.5)$ & $2.5 \pm 3.2$ & $3(25.0)$ & $2.8 \pm 8.6$ & $2(40.0)$ & $3.2 \pm 6.6$ \\
\hline Other & $6(15.8)$ & $0.8 \pm 2.1$ & $2(16.7)$ & $0.2 \pm 0.4$ & - & - \\
\hline Total, mean \pm SD & & $22.6 \pm 28.8$ & & $10.8 \pm 11.1$ & & $33.0 \pm 34.2$ \\
\hline
\end{tabular}

FP, first-time prescription; PA, pharmacy assistant; PIS, Pharmacy Information System; QT-DDI, QT drug-drug interaction; RP, repeat prescription.

in community pharmacies. Our study has shown that the implementation of an advanced CDS tool did, not significantly, reduce the proportion of QT-DDIs for which an intervention was made $(43.0-35.7 \%, p=0.14)$. However, pharmacists seemed to spend less time on the management of QT-DDIs when the CDS tool was used (6 min per QT-DDI). Overall, the pharmacists were satisfied using the tool to support the management of QT-DDIs in clinical practice.

At first, we hypothesized that an advanced CDS tool would result in more interventions, but more than $70 \%$ of the QT-DDI alerts did not require an intervention according to the CDS tool and could be considered as irrelevant. Several other studies show that specification of alert triggers by advanced clinical decision rules could decrease the alert rate up to $90 \% .{ }^{34-37}$ In total, $75 \%$ of QT-DDIs were handled with the CDS tool. Pharmacists did not comply with the flowchart of the CDS tool when the QT-DDIs included amiodarone, because they did not feel comfortable dispensing QT-DDIs with amiodarone. Therefore, if the tool is implemented in clinical practice, education on the risks of QTcprolongation and $\mathrm{TdP}$ can be useful to achieve more compliance by pharmacists.

Repeat prescriptions for chronic medication are common in primary care. However, many QT-DDIs are only relevant at the start of therapy, and are, therefore, more likely to be followed by an intervention. ${ }^{11}$ In our study, $50 \%$ of the first-time prescription QT-DDIs were followed by an intervention and $21 \%$ of the repeat-prescription QT-DDIs were followed by an intervention, as is shown in Table 7 . The CDS tool enables reassessment of repeat-prescription QT-DDIs when the condition of a patient might change during chronic treatment where a patient might become a high-risk patient.

Van der Sijs et al. showed that QT-DDI overriding rarely (33\%) results in ECG recording in a hospital setting. ${ }^{38}$ Expectedly, our study showed that in primary care this percentage is even lower $(1.7 \%)$, as ECG recording is not feasible in community pharmacies. The CDS tool does not specify management recommendations when an intervention is required, as an individualized risk assessment depending on the patient's situation by a health care professional is still important to determine the type of intervention. The combination of QTCprolonging drugs may result in potential fatal $\mathrm{TdP}$, which rarely occurs. Consensus exists that measuring the QTc-interval on the ECG is the best option to predict which patients are at risk, and the QTc is a proxy for the patient outcome. However, making ECGs for all patients filling their prescriptions in community pharmacies is not feasible and not necessary, because the QTc-prolongation risk may vary considerably among patients and QT-DDIs do not always require intervention. 
Table 7. Time for handling QT-DDIs in community pharmacies after implementation of the clinical decision support tool.

\begin{tabular}{|c|c|c|c|c|c|c|}
\hline \multirow[t]{2}{*}{ Time-management QT-DDIs } & \multicolumn{2}{|l|}{ FP, $n=40$} & \multicolumn{2}{|l|}{$\mathrm{RP}, n=4$} & \multicolumn{2}{|c|}{ Discharge, $n=1$} \\
\hline & $n(\%)$ & $\begin{array}{l}\text { Minutes } \\
\text { Mean } \pm \text { SD }\end{array}$ & $n(\%)$ & $\begin{array}{l}\text { Minutes } \\
\text { Mean } \pm \text { SD }\end{array}$ & $n(\%)$ & $\begin{array}{l}\text { Minutes } \\
\text { Mean } \pm \text { SD }\end{array}$ \\
\hline Literature & - & - & - & - & - & - \\
\hline Consult prescriber & $23(57.5)$ & $4.5 \pm 6.1$ & - & - & - & - \\
\hline Consult PA & $17(42.5)$ & $1.3 \pm 2.3$ & $2(50.0)$ & $0.7 \pm 0.6$ & 1 & 4.0 \\
\hline Documentation in PIS & $36(90.0)$ & $2.0 \pm 2.0$ & $3(75.0)$ & $3.0 \pm 2.0$ & 1 & 2.0 \\
\hline Consult patient & $17(42.5)$ & $1.8 \pm 3.4$ & $1(25.0)$ & $0.3 \pm 0.6$ & 1 & 10.0 \\
\hline Other & $8(20.0)$ & $1.7 \pm 4.1$ & $1(25.0)$ & $0.7 \pm 1.2$ & 1 & 3.0 \\
\hline Clinical rule & 37 (92.5) & $4.4 \pm 4.5$ & $3(25.0)$ & $7.0 \pm 6.9$ & 1 & 1.0 \\
\hline Total, mean \pm SD & & $15.0 \pm 16.3$ & & $11.7 \pm 7.6$ & & 20.0 \\
\hline
\end{tabular}

A strength of this study was the pre- and postdesign of the study which enables us to study the current handling of QT-DDIs. Also, the 20 community pharmacies included in this study represent the general Dutch community pharmacies according to the Dutch Foundation for Pharmaceutical Statistics (SFK) Facts and Figures of 2015.

This study also has some potential limitations. First, the tool was paper-based and was not integrated into the electronic CDS system of the community pharmacies. Consequently, the documentation of the QT-DDIs was limited because work processes in community pharmacies are fully digitalized. Only 48 forms were completed versus the 157 unique QT-DDIs generated by the CDS system in the after-period. We do realize that due to these limited completed forms our study was underpowered to make definitive conclusions regarding the tool. We hypothesize that the non-significant decrease in interventions in this study might turn into a significant decrease in interventions when performed in a larger dataset. Thereby, it should be noticed that the discriminative ability of the tool was poor, and missed errors may have occurred.

Second, apart from the criteria incorporated in step 1 of the flowchart, the tool does not stratify the various QT-DDIs. Although the QTc-prolonging drugs have different pharmacological pathways for inducing QTc-prolongation, it is relatively unknown whether combining QTc-prolonging drugs with different pharmacological pathways has an additive or synergistic effect on the extent of QTc-prolongation. ${ }^{39,40}$ Therefore, in this study we assumed the synergistic effect of the QTcprolonging drugs to be similar. Also, the number of laboratory values retrieved from the pharmacy information systems was low. In reality, this number of available laboratory values is higher because pharmacy information systems are frequently linked to information systems of GPs. In $58.1 \%$ of the included pharmacies, the pharmacy information system was linked to the GP information system, as shown in Table 1. Additionally, a potassium level of $<2.5 \mathrm{mmol} \mathrm{L}^{-1}$ scores two points according to the risk model; however, it is unlikely that an outpatient would have a value this low and any measurement this low would likely have been managed in an inpatient setting. Although the aim of the present study was to evaluate how such a tool would perform in a primary care setting, the tool was also developed for use in hospitals, and in such a setting a low potassium level may occur.

In total, four pharmacies dropped out of the study due to construction work of the pharmacy and shortages of personnel. These could potentially lead to biased results, but as these pharmacists dropped out before the implementation of the tool, this is probably not the case. 
For future perspectives, this clinical decision support tool deserves further investigation to assess its effect when it is integrated in the pharmacy information system. Such a study should be performed in large patient groups with clinically relevant endpoints such as QTc-prolongation before implementation in clinical practice can be recommended. Ideally, the system will then automatically calculate a risk score for the individual patient and only generate alerts if the risk score is $>6$, resulting in more specific alerts.

In conclusion, these results suggest that the clinical decision support tool might be an effective tool to manage QT-DDIs via a structured approach, through which a more specific advice can be given to prescribers. Also, if the condition of patients were to change during chronic treatment, the CDS tool can easily identify these potential harmful changes. Pharmacists are satisfied to use the tool and it has proven to be feasible in clinical practice. However, optimization of the tool is required before implementation in clinical practice.

\section{Acknowledgements}

The authors thank all pharmacists who participated in this research project. We also thank the SIR Institute for Pharmacy Practice and Policy (Leiden, The Netherlands) and Health Base Foundation (Houten, The Netherlands) for their contribution to this research project.

\section{Author contributions}

PvdB and TvG designed the research study. FB contributed to the conduct of the study, where FB collected data from the participating pharmacies. Data analysis was performed by FB. The results were analysed, interpreted, and discussed by FB, HvdS, PvdB, TvG. FB drafted the manuscript and all co-authors revised and approved the final version of the manuscript.

\section{Conflict of interest statement}

The authors declare that there is no conflict of interest.

\section{Funding}

The authors disclosed receipt of the following financial support for the research, authorship, and/or publication of this article: The study was supported by a grant from the Royal Dutch Pharmacists Association (Koninklijke Nederlandse
Maatschappij ter bevordering der Pharmacie, KNMP holding B.V.).

\section{ORCID iD}

Florine A. Berger (iD https://orcid.org/0000-0003 -2723-8919

\section{Supplemental material}

Supplemental material for this article is available online.

\section{References}

1. Woosley RL, Heise CW and Romero KA. QTdrugs list, www.crediblemeds.org (2008, accessed 10 October 2016).

2. Woosley RL, Romero K, Heise CW, et al. Adverse Drug Event Causality Analysis (ADECA): a process for evaluating evidence and assigning drugs to risk categories for sudden death. Drug Saf 2017; 40: 465-474.

3. Straus SM, Kors JA, De Bruin ML, et al. Prolonged QTc interval and risk of sudden cardiac death in a population of older adults. f Am Coll Cardiol 2006; 47: 362-367.

4. Moss AJ, Schwartz PJ, Crampton RS, et al. The long QT syndrome. Prospective longitudinal study of 328 families. Circulation 1991; 84: $1136-1144$.

5. Drew BJ, Ackerman MJ, Funk M, et al. Prevention of torsade de pointes in hospital settings: a scientific statement from the American heart association and the American college of cardiology foundation. Circulation 2010; 121 : 1047-1060.

6. De Bruin ML, Langendijk PN, Koopmans RP, et al. In-hospital cardiac arrest is associated with use of non-antiarrhythmic QTcprolonging drugs. Br f Clin Pharmacol 2007; 63: 216-223.

7. Roden DM. Drug-induced prolongation of the QT interval. N Engl F Med 2004; 350: 1013-1022.

8. Schwartz PJ and Woosley RL. Predicting the unpredictable: drug-induced QT prolongation and torsades de pointes. $\mathcal{F} \mathrm{Am} \mathrm{Coll} \mathrm{Cardiol} \mathrm{2016;}$ 67: 1639-1650.

9. Nachimuthu S, Assar MD and Schussler JM. Drug-induced QT interval prolongation: mechanisms and clinical management. Ther $A d v$ Drug Saf 2012; 3: 241-253.

10. Heringa M, Floor-Schreudering A, Tromp PC, et al. Nature and frequency of drug therapy 
alerts generated by clinical decision support in community pharmacy. Pharmacoepidemiol Drug Saf 2016; 25: 82-89.

11. Buurma H, De Smet PA and Egberts AC. Clinical risk management in Dutch community pharmacies: the case of drug-drug interactions. Drug Saf 2006; 29: 723-732.

12. Buurma H, Schalekamp T, Egberts AC, et al. Compliance with national guidelines for the management of drug-drug interactions in Dutch community pharmacies. Ann Pharmacother 2007; 41: 2024-2031.

13. Isaac T, Weissman JS, Davis RB, et al. Overrides of medication alerts in ambulatory care. Arch Intern Med 2009; 169: 305-311.

14. Nanji KC, Slight SP, Seger DL, et al. Over rides of medication-related clinical decision support alerts in outpatients. $\mathcal{F}$ Am Med Inform Assoc 2014; $21: 487-491$.

15. Ojeleye O, Avery A, Gupta V, et al. The evidence for the effectiveness of safety alerts in electronic patient medication record systems at the point of pharmacy order entry: a systematic review. $B M C$ Med Inform Decis Mak 2013; 13: 69.

16. Phansalkar S, van der Sijs H, Tucker AD, et al. Drug-drug interactions that should be noninterruptive in order to reduce alert fatigue in electronic health records. $\mathcal{F} \mathrm{Am} \mathrm{Med}$ Inform Assoc 2013; 20: 489-493.

17. Seidling HM, Klein U, Schaier M, et al. What, if all alerts were specific - estimating the potential impact on drug interaction alert burden. Int $\mathcal{F}$ Med Inform 2014; 83: 285-291.

18. Seidling HM, Phansalkar S, Seger DL, et al. Factors influencing alert acceptance: a novel approach for predicting the success of clinical decision support. F Am Med Inform Assoc 2011; 18: 479-484.

19. McCoy AB, Thomas EJ, Krousel-Wood M, et al. Clinical decision support alert appropriateness: a review and proposal for improvement. Ochsner $\mathcal{F}$ 2014; 14: 195-202.

20. Payne TH, Hines LE, Chan RC, et al. Recommendations to improve the usability of drug-drug interaction clinical decision support alerts. F Am Med Inform Assoc 2015; 22: 1243-1250.

21. Berger FA, van der Sijs H, Becker ML, et al. Development and validation of a tool to assess the risk of QT drug-drug interactions in clinical practice. BMC Med Inform Decis Mak 2020; 20: 171.
22. Berger FA, van der Sijs $H$, van Gelder $T$, et al. Comparison of two algorithms to support medication surveillance for drug-drug interactions between QTc-prolonging drugs. Int $\mathcal{F}$ Med Inform 2020; 145: 104329.

23. Matsukura S, Nakamura Y, Cao X, et al. Antiatrial fibrillatory versus proarrhythmic potentials of amiodarone: a new protocol for safety evaluation in vivo. Cardiovasc Toxicol 2017; 17: 157-162.

24. Biewenga J, Keung C, Solanki B, et al. Absence of QTc prolongation with domperidone: a randomized, double-blind, placebo- and positivecontrolled thorough QT/QTc study in healthy volunteers. Clin Pharmacol Drug Dev 2015; 4: 41-48.

25. Boyce MJ, Baisley KJ and Warrington SJ. Pharmacokinetic interaction between domperidone and ketoconazole leads to QT prolongation in healthy volunteers: a randomized, placebo-controlled, double-blind, crossover study. Br f Clin Pharmacol 2012; 73: 411-421.

26. Wang SM and Pae CU. How much to worry about the FDA warning in the use of citalopram? Expert Rev Neurother 2013; 13: 883-886.

27. Leonard CE, Bilker WB, Newcomb C, et al. Antidepressants and the risk of sudden cardiac death and ventricular arrhythmia. Pharmacoepidemiol Drug Saf 2011; 20: 903913.

28. Macht M, Mull AC, McVaney KE, et al. Comparison of droperidol and haloperidol for use by paramedics: assessment of safety and effectiveness. Prehosp Emerg Care 2014; 18: 375-380.

29. Blom MT, Bardai A, van Munster BC, et al. Differential changes in QTc duration during in-hospital haloperidol use. PLoS One 2011; 6: e23728.

30. Duprey MS, Al-Qadheeb N, Roberts R, et al. The use of low-dose IV haloperidol is not associated with QTc prolongation: post hoc analysis of a randomized, placebo-controlled trial. Intensive Care Med 2016; 42: 1818-1819.

31. Brooke J. SUS: A quick and dirty usability scale. In: Jordan PW, Thomas B, Weerdmeester BA, et al. (eds) Usability evaluation in industry. London: Taylor and Francis, 1996.

32. Bangor A, Kortum PT and Miller JT. An empirical evaluation of the system usability scale. Int F Hum-Comput Int 2008; 24: 574-594. 
33. Aaron B, Philip K and James M. Determining what individual SUS scores mean: adding an adjective rating scale. $\mathcal{F}$ Usability Studies 2009; 4: $114-123$.

34. Helmons PJ, Suijkerbuijk BO, Nannan Panday $\mathrm{PV}$, et al. Drug-drug interaction checking assisted by clinical decision support: a return on investment analysis. F Am Med Inform Assoc 2015; 22: 764-772.

35. Eppenga WL, Derijks HJ, Conemans JM, et al. Comparison of a basic and an advanced pharmacotherapy-related clinical decision support system in a hospital care setting in the Netherlands. F Am Med Inform Assoc 2012; 19: 66-71.

Visit SAGE journals online journals.sagepub.com/ home/taw

@SAGE journals

36. Czock D, Konias M, Seidling HM, et al. Tailoring of alerts substantially reduces the alert burden in computerized clinical decision support for drugs that should be avoided in patients with renal disease. 7 Am Med Inform Assoc 2015; 22: 881-887.

37. Heringa $M$, van der Heide A, Floor-Schreudering A, et al. Better specification of triggers to reduce the number of drug interaction alerts in primary care. Int F Med Inform 2018; 109: 96-102.

38. van der Sijs H, Kowlesar R, Klootwijk AP, et al. Clinically relevant QTc prolongation due to overridden drug-drug interaction alerts: a retrospective cohort study. Br f Clin Pharmacol 2009; 67: 347-354.

39. Meid AD, von Medem A, Heider D, et al. Investigating the additive interaction of QT-prolonging drugs in older people using claims data. Drug Saf 2017; 40: 133-144.
40. Alexandrou AJ, Duncan RS, Sullivan A, et al. Mechanism of hERG $\mathrm{K}+$ channel blockade by the fluoroquinolone antibiotic moxifloxacin. $\mathrm{Br} \mathcal{F}$ Pharmacol 2006; 147: 905-916. 